\title{
ALTERAÇÕES EM CARACTERÍSTICAS QUÍMICAS DE SOLOS DA REGIÃo AMAZÔNICA PELA INTRODUÇÃo DE PASTAGENS ${ }^{1}$
}

\author{
Regina M. LONGO', Carlos R. ESPÍNDOLA²
}

RESUMO - O presente trabalho objetiva avaliar os impactos decorrentes da remoção da cobertura original e seu posterior uso com pastagem, tendo como atributos principais de análises, as modificações nas propriedades químicas do solo. As amostras foram retiradas na região de Porto Velho/RO, junto a Embrapa (Empresa Brasileira de Pesquisa Agropecuária) em latossolo vermelhoamarelo. Estas foram coletadas ao longo de uma transeção contendo 24 pontos, 12 sob vegetação natural (floresta) e 12 sob pastagem plantada à aproximadamente 15 anos em dois horizontes distintos: superficial e subsuperficial. Os atributos químicos mostraram diferenças significativas na substituição floresta-pastagem para $\mathrm{V} \%, \mathrm{CTC}, \mathrm{H}+\mathrm{Al}, \mathrm{N}$-total e $\mathrm{C}$-orgânico, sendo os maiores valores encontrados no horizonte superficial do solo de floresta, ocorrendo também uma estocagem mais nítida de $\mathrm{P}, \mathrm{Ca}, \mathrm{Mg}$ e maiores valores de CTC, N-total e C-orgânico no horizonte superficial das duas situações estudadas (vegetação natural e pastagem), evidenciando o efeito da cobertura vegetal sobre o conteúdo e distribuição dos componentes orgânicos e minerais em solos tropicais.

Palavras-chave: impacto ambiental, Rondônia, cobertura vegetal

\section{Modifications in Amazon Soil's Chemical Properties Caused by the Introduction of Pasture}

SUMMARY - The aim of the present work is to evaluate the effects of the human intervention, through the introduction of pasture, in some chemical properties of the Amazon soil. This research was installed in Porto Velho, State of Rondônia - Brazil, located vicinity of the Empresa Brasileira de Pesquisa Agropecuária (Embrapa). The samples were accomplished in areas of primary vegetation and planted pasture (about 15 years), obeying a line with 24 points each 10 meters (12 under natural vegetation and 12 under pasture) in two horizons (superficial and undersuperficial). The results obtained for chemical compounds showed significant between forest and pasture to $\mathrm{V} \%, \mathrm{CEC}, \mathrm{C}+\mathrm{Al}, \mathrm{N}$-total and $\mathrm{C}$-organic, mainly in the superficial horizon of the original vegetable. The higher organic compounds ( $\mathrm{N}$-total, $\mathrm{C}$-organic) and $\mathrm{P}, \mathrm{Ca} \mathrm{e} \mathrm{Mg}$, in the superficial horizon of natural vegetation, wintry in Amazon Forest were observed.

Key-words: environmental impact, Rondônia, natural vegetation

\section{INTRODUÇÃO}

O Brasil é considerado um dos países com flora mais abundantes da Terra, contando com aproximadamente 60.000 espécies, das cerca de 220.000 reconhecidas entre as Angiospermas. Tal fato certamente está relacionado à vasta extensão ter- ritorial (mais de $8.500 .000 \mathrm{~km}^{2}$ ) e à grande diversidade de climas, solos e condições geomorfológicas, as quais produzem enorme variedade de tipos de vegetação (Giulietti, 1992).

Dentro do Território Nacional, a Amazônia Legal abrange cerca de 450 milhões de hectares, com a floresta tropical úmida estendendo-se por 280

\footnotetext{
'Trabalho desenvolvido com suporte financeiro da Fundação de Amparo à Pesquisa do Estado de São Paulo (FAPESP)

${ }^{2}$ Aluna de Doutorado da Faculdade de Engenharia Agrícola - UNICAMP - Cidade Universitária Zeferino Vaz, Caixa Postal 6011, Barão Geraldo, Campinas - SP, Brasil, CEP: 13081-490. Email: longo@agr.unicamp.br

${ }^{3}$ Prof. Titular da Faculdade de Engenharia Agricola - UNICAMP
} 
milhões destes, ao lado da floresta aberta ou de transição, já bastante alterada pela ação antrópica na expansão da fronteira agrícola nas regiões do cerrado e dos campos naturais (Cunha, 1989). Embora as estimativas sobre taxa de ocupação sejam bastante controvertidas, certo é que o total da área desflorestada tem crescido exponencialmente, sendo a pecuária uma das forma mais expressivas de uso da terra (Serrão \& Toledo, 1990). O Estado de Rondônia, com uma com uma área de $238.378,7$ $\mathrm{km}^{2}$, apresenta cobertura vegetal típica da região amazônica. Apesar da extensa cobertura vegetal do Estado, cerca de $8 \%$ da floresta já foram completamente destruídos, em função de empreendimentos diversos, dentre os quais a construção da Usina Hidroelétrica de Samuel, atividades de assentamento do Incra e a grande expansão das fronteiras agropecuárias. A taxa de ocupação humana e a degradação ambiental têm sido mais altas nas últimas décadas, quando comparadas àquelas de outros estados (Cunha, 1989; Nascimento \& Homma, 1984; Prance, 1987; Serrão et al., 1979; Serrão \& Toledo, 1990).

O grau de alteração provocado pela intervenção humana no sistema natural pode ser avaliado, dentre outros critérios, pela modificação em alguns atributos do solo. Quando se comparam as condições de um solo, sob sua cobertura original, com aquelas do qual esta foi retirada, ou onde houve a implantação de uma cultura, em geral é possivel notar, com maiores ou menores evidências, as modificações e os danos ocorridos.

$\mathrm{O}$ ciclo de nutrientes entre a floresta e o solo é quase que fechado. A constante decomposição do material orgânico e a pequena perda por lixiviação permitem

0 desenvolvimento de uma floresta luxuriante, sem sintomas de deficiências nutricionais, em solos com baixa fertilidade natural (Demattê, 1988; Nortcliff \& Thornes, 1982; Sanches, 1976). A biomassa total de uma floresta varia entre 100 a $400 \mathrm{t} /$ ha de matéria seca, com a vegetação natural exercendo grande influência sobre o conteúdo de nutrientes disponíveis no solo (Sanches, 1976).

De acordo com Melo et al. (1988), quando um solo virgem é submetido ao cultivo estabelece-se uma nova ordem de condições que, regra geral, resulta numa queda do teor de muitos elementos. Esta, além de mais intensa nos primeiros anos, é continua, até que se estabeleça novo equilibrio entre adições e perdas. Segundo os mesmos autores, após o início do cultivo do solo em condições de clima temperado, o novo teor de equilíbrio de nitrogênio é alcançado somente em 60 a 100 anos. Já em regiões de climas tropical e subtropical esse tempo deve ser bem menor. Segundo Cravo \& Smyth (1997), a ordem de ocorrência de limitações nutricionais no cultivo contínuo e queima da vegetação, em floresta equatorial úmida, foi de fósforo, seguido de potássio, acidez, nitrogênio e manganês. 
Desta forma, o presente trabalho tem por objetivo avaliar os efeitos da introdução de pastagens em ambiente de floresta sobre determinados parâmetros químicos do solo na região de Porto Velho junto à Embrapa/RO. Com tais resultados esperou-se fornecer alguns subsídios técnicos à discussão sobre a manutenção deste ecossistema ou, na sua impossibilidade, que se proceda a sua ocupação de maneira racional.

\section{MATERIAL E MÉTODOS}

As amostragens de solo foram realizadas em áreas de pesquisa da Empresa Brasileira de Pesquisa Agropecuária (Embrapa), em latossolo vermelho-amarelo, no Município de Porto Velho/RO, numa seqüência pasto-floresta. A temperatura média anual da região foi de $25,5^{\circ} \mathrm{C}$, com uma precipitação de $2200 \mathrm{~mm}$, apresentado um periodo de seca durante os meses de maio a setembro e a vegetação natural predominante foi classificada como "floresta tropical" (Radambrasil, 1984).

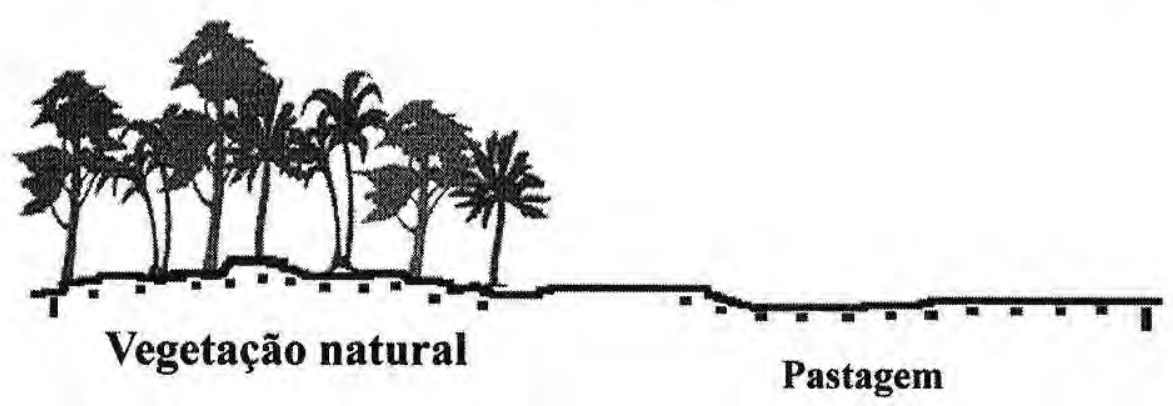

$245 \mathrm{~m}$
No local foram selecionadas áreas de vegetação primária e de pastagem plantada. No caso da pastagem, o histórico da região demonstrou que a área em estudo foi desmatada em meados de 1970 , com o decorrer dos anos tornou-se "capoeira" e a partir de 1984 a pastagem foi recuperada, fazendo calagem e adução e com o plantio de Brachiaria brizanta (Brizantão).

As amostras foram coletadas ao longo de uma transecção, com vinte e quatro pequenas trincheiras de $60 \mathrm{~cm}$ de profundidade, espaçadas de $10 \mathrm{em}$ 10 metros (12 sob pastagem e $12 \mathrm{sob}$ vegetação natural), seguindo-se recomendações sugeridas por Sparovek (1993) para situações análogas, conforme esquema da Figura 1.

As amostras foram retiradas dos horizontes superficiais e subsuperficiais nas duas situações estudadas, cujas profundidades variaram em função das peculiaridades existentes. Em cada ponto de coleta foram abertas

Figura 1. Esquema de amostragem utilizado para a coleta de dados 
pequenas trincheiras, de aproximadamente $60 \mathrm{~cm}$ de profundidade, para uma sucinta descrição pedológica. De maneira geral, as profundidades de coletas foram de $0-15$ e $0-8 \mathrm{~cm}$ no horizonte superficial do solo sob floresta e pastagem, respectivamente, e de 15 45 e $8-20 \mathrm{~cm}$ para o horizonte subsuperficial.

Cada três pontos de coleta, ao longo da transecção, foram tomados como subparcelas. As três repetições de cada subparcela (cada ponto de amostragem) foram comparadas pela análise de variância (teste $\mathrm{F}$ ) enquanto a comparação das médias foi feita pelo teste Ducan com 5\% de significância. As análises de variância foram realizadas em esquema de parcelas subdivididas, visando verificar a ocorrência ou não de diferenças significativas entre as parcelas (vegetação natural e pastagem) e entre as subparcelas (posição de amostragem).

As amostras de terra fina seca ao ar foram submetidas a análises químicas, determinando-se: $\mathrm{pH}$ em água; teor de matéria orgânica por oxidação pelo $\mathrm{H}_{2} \mathrm{SO}_{4}$ e por quantificação colorimétrica; fósforo disponivel $(\mathrm{P})$ e teores de potássio $(\mathrm{K})$, cálcio (Ca) e magnésio $(\mathrm{Mg})$, extraidos pela resina de troca iônica, sendo o P quantificado por colorimetria e o $\mathrm{K}, \mathrm{Ca}$ e $\mathrm{Mg}$ por espectrofotometria de absorção atômica; teor de aluminio (Al) extraido pelo $\mathrm{KCl} 1 \mathrm{~N}$ e determinado por titulação ácido-base; $\mathrm{T}=$ capacidade de troca de cátiosn e V= saturação por base, foram calculados. A descrição detalhada dos métodos analiticos empregados podem ser vistos em Raij (1987). O N-total foi determinado pelo método Kjeldahl, conforme descrito por Ferreira et al. (1974) e Melo (1974). Foi utilizado o método de Tiurin (oxidação por via úmida), conforme descrito em Dabin (1976) para a determinação do carbono orgânico. A relação $\mathrm{C} / \mathrm{N}$ foi obtida através da relação entre o teor de Carbono orgânico e Nitrogênio total para cada amostra em estudo.

\section{RESULTADOS E DISCUSSÃO}

Os resultados obtidos para o $\mathrm{pH}$ (Tab. 1) mostraram um solo ácido e, segundo Smyth (1996), a predominância de solos ácidos em terra firme coloca a acidez e a toxidez de aluminio entre as três mais freqüentes restrições de fertilidade de solos da Amazônia. A pastagem apresentou valores ligeiramente superiores aos da floresta. Falesi (1976), estudando cronoseqüências de pastagens no Pará e no Mato Grosso, encontrou que o $\mathrm{pH}$, a CTC e a saturação de bases da camada superficial do solo permaneciam elevadas em todas as áreas de pastagem. Estes resultados sugerem que, desde que seja evitada uma erosão mais intensa da área por meio do manejo mais adequado da pastagem, os cátions podem ser retidos.

Em relação aos valores de porcentagem de saturação de bases (V\%), soma de bases (S) e capacidade 
Tabela 1. $\mathrm{pH}, \% \mathrm{MO}, \mathrm{V} \%, \mathrm{~S}$ e CTC em solos do Cerrado e da Amazônia (*).

\begin{tabular}{|c|c|c|c|c|c|c|c|c|}
\hline \multirow[t]{2}{*}{ Horizontes } & \multicolumn{5}{|c|}{ Floresta } & \multicolumn{3}{|c|}{ Pastagem } \\
\hline & 1 & 2 & 3 & 4 & 5 & 6 & 7 & 8 \\
\hline \multicolumn{9}{|c|}{$\mathrm{pH}\left(\mathrm{CaCl}_{2}\right)$} \\
\hline $\mathrm{Ao} / \mathrm{A}$ & $3,6 a$ & $3,5 a$ & $3,8 a$ & $3,6 a$ & $4,1 \mathrm{a}$ & $3,9 a$ & $4,1 \mathrm{a}$ & $4,4 a$ \\
\hline$A B$ & $4,0 \mathrm{~b}$ & $3,9 b$ & $3,9 b$ & $3,9 b$ & $4,1 b$ & $4,0 b$ & $4,2 b$ & $4,5 b$ \\
\hline \multicolumn{9}{|c|}{ Matéria orgânica (\%) } \\
\hline $\mathrm{Ao} / \mathrm{A}$ & $6,8 a$ & $6,7 a$ & $4,6 a$ & $4,7 a$ & $4,2 a$ & $4,2 \mathrm{a}$ & $4,1 \mathrm{a}$ & $4,2 a$ \\
\hline $\mathrm{AB}$ & $2,7 b$ & $3,3 b$ & $3,2 b$ & $3,4 b$ & $2,5 b$ & $2,9 b$ & $2,4 b$ & $2,7 b$ \\
\hline \multicolumn{9}{|c|}{ V (\%) } \\
\hline $\mathrm{Ao} / \mathrm{A}$ & $1 a(* *)$ & $2 a$ & $4 a$ & $3 a$ & $12 b$ & $5 a$ & $10 b$ & $14 b$ \\
\hline$A B$ & $2 a$ & $2 a$ & $2 a$ & $2 a$ & $9 b$ & $4 a$ & $4 \mathrm{~b}$ & $6 b$ \\
\hline \multicolumn{9}{|c|}{$\mathrm{S}\left(\mathrm{meq} / 100 \mathrm{~cm}^{3}\right)$} \\
\hline $\mathrm{Ao} / \mathrm{A}$ & $0,3 a$ & $0,3 a$ & $0,6 a$ & $0,4 a$ & $1,3^{\mathrm{a}}$ & 0,5 & $1,0 \mathrm{a}$ & $2,0 a$ \\
\hline$A B$ & $0,2 a$ & $0,2 a$ & $0,3 a$ & $0,2 a$ & $0,6^{\mathrm{a}}$ & $0,5 a$ & $0,3 a$ & $1,1 \mathrm{a}$ \\
\hline \multicolumn{9}{|c|}{ CTC $\left(\mathrm{meq} / 100 \mathrm{~cm}^{3}\right)$} \\
\hline Ao/A & $18,2 a$ & $18,3 a$ & $14,2 a$ & $15,9 a$ & $12,1 b$ & $12,7 b$ & $10,3 b$ & $9,8 b$ \\
\hline$A B$ & $9,5 c$ & $10,8 c$ & $10,3 c$ & $11,5 \mathrm{c}$ & $8,3 d$ & $10,2 d$ & $7,3 d$ & $8,2 d$ \\
\hline
\end{tabular}

(") Média de três repetiçōes

(*) Diferenças significativas (Ducan 5\%) indicadas por letras distintas

de troca catiônica (CTC), (Tab. 1), observou-se que o solo sob floresta apresentou, em média, altos valores de $\mathrm{CTC}^{\prime}$ no horizonte superficial, provavelmente, em função do elevado conteúdo de matéria orgânica existente neste horizonte e com teor moderado no horizonte subsuperficial. Por outro lado, foram baixos os valores de soma de bases trocáveis e de V\% em ambos horizontes.

Assim, além da participação da matéria orgânica, pode-se deduzir que a alta CTC foi dada por expressivas contribuição de $\mathrm{H}^{+} \mathrm{e} \mathrm{Al}^{+3}$, elementos esses ligados à acidez do solo. O solo sob pastagem apresenta valores moderados de CTC, e baixo de V\% e S. Okali (1992) chamou a atenção para a pequena atividade das argilas $1: 1$ que dominam os ambientes dos trópicos úmidos, ficando a fertilidade dos solos, consequentemente, garantida quase que inteiramente pela adição de matéria orgânica, o que se aplica perfeitamente às condições

I Intervalo de classificação utilizado - Mello et al. (1988) 
estudadas, com o predomínio absoluto da caulinita acompanhada da gibsita.

As distribuições dos teores de fósforo $(\mathrm{P})$, potássio $(\mathrm{K})$, cálcio $(\mathrm{Ca})$, magnésio $(\mathrm{Mg})$ e $\mathrm{H}+\mathrm{Al}$ podem ser observadas na Tabela 2. Pela análise dos dados pode-se verificar que os teores de fósforo apresentaram-se de baixo a médio' no horizonte superficial, tanto sob vegetação natural quanto sob pastagem, não havendo diferenças significativas entre os manejos estudados. No horizonte subsuperficial esses teores foram sempre baixos. Resultado diferentes foram observados por alguns autores (Falesi, 1976; Nye \& Greeland, 1964; Seubert et al. 1977) que observaram, após a derrubada e queima da floresta, que o fósforo geralmente aumenta em decorrência da deposição de cinzas e material vegetal decomposto. No entanto, diferentemente de outros elementos que, geralmente, são

Tabela 2. Análise de fertilidade em solos do Cerrado e da Amazônia (*).

\begin{tabular}{|c|c|c|c|c|c|c|c|c|}
\hline \multirow[t]{2}{*}{ Horizontes } & \multicolumn{4}{|c|}{ Floresta } & \multicolumn{4}{|c|}{ Pastagem } \\
\hline & 1 & 2 & 3 & 4 & 5 & 6 & 7 & 8 \\
\hline \multicolumn{9}{|c|}{$\mathrm{P}\left(\mathrm{ug} / \mathrm{cm}^{3}\right)$} \\
\hline $\mathrm{Ao} / \mathrm{A}$ & $8 a^{(*)}$ & $10 a$ & $8 a$ & $9 a$ & $9 a$ & $9 a$ & $9 a$ & $9 a$ \\
\hline$A B$ & $5 b$ & $5 b$ & $6 b$ & $7 b$ & $4 b$ & $6 b$ & $5 b$ & $5 b$ \\
\hline \multicolumn{9}{|c|}{$\mathrm{K}\left(\mathrm{meq} / 100 \mathrm{~cm}^{3}\right)$} \\
\hline $\mathrm{Ao} / \mathrm{A}$ & $0,05 a$ & $0,06 a$ & $0,08 \mathrm{a}$ & $0,05 a$ & $0,04 a$ & $0,02 a$ & $0,05 a$ & $0,09 a$ \\
\hline$A B$ & $0,03 a$ & $0,03 a$ & $0,04 a$ & $0,02 \mathrm{a}$ & $0,01 \mathrm{a}$ & $0,02 a$ & $0,01 a$ & $0,01 \mathrm{a}$ \\
\hline \multicolumn{9}{|c|}{$\mathrm{Ca}\left(\mathrm{meq} / 100 \mathrm{~cm}^{3}\right)$} \\
\hline $\mathrm{Ao} / \mathrm{A}$ & $0,1 \mathrm{a}$ & $0,1 a$ & $0,2 a$ & $0,2 a$ & $1,0 a$ & $0,4 a$ & $0,7 a$ & $2,6 b$ \\
\hline$A B$ & $0,1 a$ & $0,1 a$ & $0,1 \mathrm{a}$ & $0,1 \mathrm{a}$ & $0,5 a$ & $0,3 a$ & $0,2 a$ & $1,9 b$ \\
\hline \multicolumn{9}{|c|}{$\mathrm{Mg}\left(\mathrm{meq} / 100 \mathrm{~cm}^{3}\right)$} \\
\hline $\mathrm{Ao} / \mathrm{A}$ & $0,1 \mathrm{a}$ & $0,1 \mathrm{a}$ & $0,3 a$ & $0,2 a$ & $0,3 a$ & $0,2 a$ & $0,3 a$ & $0,6 a$ \\
\hline$A B$ & $0,1 a$ & $0,1 \mathrm{a}$ & $0,1 \mathrm{a}$ & $0,1 a$ & $0,1 a$ & $0,1 a$ & $0,1 a$ & $0,2 a$ \\
\hline \multicolumn{9}{|c|}{$\mathrm{H}+\mathrm{Al}\left(\mathrm{meq} / 100 \mathrm{~cm}^{3}\right)$} \\
\hline $\mathrm{Ao} / \mathrm{A}$ & $17,9 a$ & $18,0 \mathrm{a}$ & $13,6 a$ & $15,6 a$ & $11,7 b$ & $12,2 b$ & $9,3 b$ & $6,9 b$ \\
\hline$A B$ & $9,2 \mathrm{c}$ & $10,0 \mathrm{c}$ & $10,0 \mathrm{c}$ & $11,3 c$ & $7,7 d$ & $9,8 d$ & $6,9 d$ & $4,6 d$ \\
\hline
\end{tabular}


mantidos em niveis mais ou estáveis sob pastagem, o $\mathrm{P}$ disponivel na camada superficial do solo normalmente, cai drasticamente com o tempo, após o estabelecimento desta (Falesi, 1976).

Os teores de potássio não apresentaram diferenças significativas para manejo (vegetação natural e pastagem). Em profundidade, mostraram-se sempre baixos, onde teores mais elevados foram observados nas camadas superficiais, embora essa diferença, não tenha sido significativa. Comportamento similar foi observado para o magnésio. Segundo Demattê (1988) após a queima e a introdução de práticas agrícolas, os teores de potássio trocável aumentam, não sendo porém este aumento duradouro. Resultados similares foram encontrados para $\mathrm{Ca}$ e $\mathrm{Mg}$, sendo esses resultados provavelmente devidos a intensa lixiviação que ocorre em solos sob ecossistemas tropicais (Primavesi, 1987).

Os teores de cálcio revelaram-se baixos $^{1}$, com o solo sob vegetação de floresta apresentando os menores valores absolutos, não havendo diferença significativa ao nível estudado. Segundo Mello et al. (1988), em regiões úmidas os solos derivados de rochas calcárias são freqüentemente ácidos nas camadas superficiais, devido à remoção de cátions pela lavagem excessiva. A água contendo $\mathrm{CO}_{2}$ dissolvido atravessa o perfil, o ácido carbônico formado desloca o $\mathrm{Ca}$ (e outros cátions) do complexo coloidal e paulatinamente o terreno vai se euxarindo de bases e acidificando-se, o que justifica, provavelmente, os baixos teores de $\mathrm{Ca}$ encontrados.

Os valores de $\mathrm{H}+\mathrm{Al}$ revelaram diferenças significativas entre manejos e profundidades estudados. Os maiores valores foram observados no solo sob vegetação natural, indicando que estes são muito ácidos e podem apresentar toxidez ao $\mathrm{Al}$, mostrando, desta forma, uma restrição à fertilidade, resultados esses que concordam com as observações de Smyth (1996).

Os teores de N-total (Tab, 3) apresentaram diferenças significativas em profundidade e manejo (vegetação natural e pastagem). O solo sob vegetação natural apresentou teores mais elevados, nas duas profundidades estudadas, em consonância com a exuberância da floresta, aliada às condições climáticas de maior umidade e as condições que favorecem o desenvolvimento vegetativo.

Os horizontes superficiais apresentaram as maiores concentrações de nitrogênio, concordando com constatações obtidas por Cerri (1989), em solos da Bacia Amazônica, de que 41,8\% do $\mathrm{N}$-total encontraram-se estocados nos primeiros $20 \mathrm{~cm}$ do solo, devido, ao maior acúmulo de material orgânico nos horizontes superficiais.

$\mathrm{O}$ carbono orgânico (Tab. 3) revelou comportamento similar ao do $\mathrm{N}$-Total, observando-se que o solo sob 
Tabela 3. N-total, C-orgânico e relação $\mathrm{C} / \mathrm{N}$ em solos do Cerrado e da Amazônia (*).

\begin{tabular}{|c|c|c|c|c|c|c|c|c|}
\hline \multirow[t]{2}{*}{ Horizontes } & \multicolumn{5}{|c|}{ Floresta } & \multicolumn{3}{|c|}{ Pastagem } \\
\hline & 1 & 2 & 3 & 4 & 5 & 6 & 7 & 8 \\
\hline \multicolumn{9}{|c|}{$\mathrm{N}$-Total $\left(\mathrm{g} \cdot \mathrm{Kg}^{-1}\right)$} \\
\hline $\mathrm{Ao} / \mathrm{A}$ & $3,0 \mathrm{a}$ & $2,9 a$ & $2,5 a$ & $2,8 a$ & $2,1 b$ & $2,0 \mathrm{~b}$ & $2,1 b$ & $2,6 b$ \\
\hline$A B$ & $1,7 c$ & $1,8 \mathrm{c}$ & $1,6 \mathrm{c}$ & $1,8 \mathrm{c}$ & $1,3 d$ & $1,3 d$ & $1,5 \mathrm{~d}$ & $1,8 d$ \\
\hline \multicolumn{9}{|c|}{ C-orgânico $\left(\mathrm{g} . \mathrm{Kg}^{-1}\right)$} \\
\hline $\mathrm{Ao} / \mathrm{A}$ & $25,8 a$ & $20,5 a$ & $21,5 a$ & $18,7 a$ & $18,3 b$ & $17,5 b$ & $18,5 b$ & $14,4 b$ \\
\hline$A B$ & $17,2 \mathrm{c}$ & $15,8 \mathrm{c}$ & $15,9 c$ & $14,9 c$ & $13,9 d$ & $12,5 d$ & $13,3 d$ & $12,3 d$ \\
\hline \multicolumn{9}{|c|}{ Relação C/N } \\
\hline $\mathrm{Ao} / \mathrm{A}$ & $9 \mathrm{a}^{(")}$ & $7 a$ & $9 a$ & $7 a$ & $9 a$ & $9 a$ & $9 a$ & $5 a$ \\
\hline$A B$ & $10 a$ & $9 a$ & $10 a$ & $8 a$ & $11 a$ & $10 a$ & $5 a$ & $7 a$ \\
\hline
\end{tabular}

(*) Média de 3 repetiçőes

vegetação natural apresentou, de maneira geral, teores mais elevados deste elemento nas duas profundidades analisadas. A comparação entre vegetação natural e pastagem revelou diferenças significativas, sendo observada uma queda gradual nos teores de Corgânico ao se passar da primeira situação para a segunda, concordando com resultados de Moraes (1991), que observou que, de maneira geral, o carbono e o nitrogênio foram bastante vulneráveis ao cultivo por estarem concentrados na camada superficial do solo. Uma série de estudos foram realizados, principalmente na região de regime ústico de umidade (sul do Pará), indicando algumas evidências no sistema de manejo para pastagens (Baena, 1977; Falesi, 1976; Hecht, 1982; Serrão et al., 1979). Diversos fatores foram salientados para justificar a queda de produtividade das pastagens e, consequentemente, queda no fornecimento de material orgânico: baixa fertilidade natural das terras; baixa qualidade das pastagens; gramineas inadequadas para a região; pragas e doenças e sistemas inadequados de manejo.

Em relação à implantação de pastagem, alguns autores (Cerri et al., 1992; Choné et al., 1991) demostraram que esta pode não só recuperar os teores de carbono do solo, (situação de floresta) como, em alguns casos superá-lo; os resultados ora obtidos discordaram dessa afirmação, provavelmente devido a idade das pastagens.

Em profundidade, verificou-se que, de maneira geral, o teor de Corgânico foi maior no horizonte super- 
ficial, como também observado por Moraes (1984) o qual constatou que o conteúdo de carbono e nitrogênio em solos da Bacia Amazônica encontrou-se concentrados, principalmente, na camada entre $0 \mathrm{e}$ $20 \mathrm{~cm}$.

A relação $\mathrm{C} / \mathrm{N}$ apresentou um comportamento diferenciado daquele analisado isoladamente para os teores de N-Total e C-orgânico. De maneira geral, as proporções revelaram variações pontuais, não havendo diferenças significativas entre as médias, sendo os maiores valores observados nas amostras coletadas no horizonte subsuperficial.

\section{CONCLUSÕES}

De acordo com os resultados obtidos pode-se verificar que, as mudanças na cobertura vegetal original, no sentido vegetação natural-pastagem, foram acompanhadas por uma diminuição nos teores de N-total, Corgânico indicando que, de maneira geral, estes parâmetros foram bastante vulneráveis ao cultivo, principalmente por estarem concentrados na camada superficial do solo. Já a análise de fertilidade não mostrou efeitos de modificações pela mudança na cobertura vegetal original, uma vez que a dinâmica dos nutrientes nesses solos não parece ter sido alterada por tais práticas. Em profundidade, houve uma estocagem mais nitida de $\mathrm{P}, \mathrm{Ca}, \mathrm{Mg}$, maiores valores de T, N-total, Corgânico, nos horizonte superficiais, nas duas condições de manejo estudadas (vegetação natural e pastagem.

\section{Bibliografia citada}

Baena, A.R.O, 1977. The effect of pasture (Panicum maximum) on the chemical composition of the soil after clearing and burning a typical tropical highland forest. Ames, lowa, lowa Sate University, (M.S. Thesis) 164p.

Cerri, C.C. 1989. Dinâmica da matéria orgânica em solos de pastagem. Anais Simpósio sobre ecossistemas de pastagem. p. 135-147.

Cerri, C.C.; Moraes, J.F.L., Volkoff, B. 1992. Dinâmica do carbono orgânico em solos vinculados à pastagens da Amazônia brasileira. Investigation Agrária, Madrid, 1(1): 95-102.

Choné, T.; Andreux, F.; Correa, J.C.; Volkoff, B., Cerri, C.C. 1991. Changes in organic matter in an oxisol from the central Amazon forest during eight years as pasture, determined by ${ }^{13} \mathrm{C}$ isotopic composition. In: Berthelin, J. ed. Diverssity of environmental biogeochemistry. Amsterdam, Elsevier, p. 397-405.

Cravo, M.S.; Smyth, T.J. 1997. Manejo sustentado da fertilidade de um latossolo da Amazônia central sob cultivos sucessivos. Rev. Bras. Ci. Solo, 21(3): 240-273.

Cunha, R.P. 1989. Deforestation estimates though remote sensing: the state of the art in the Legal Amazon. Annais Symposium - Amazon: facts, problems and solutions. São Paulo, University of São Paulo, p 240-273.

Dabin, B. 1976. Curso sobre matéria orgânica do solo. Parte 1. Análise dos compostos húmicos do solo. Piracicaba, CENA, $115 \mathrm{p}$.

Demattê, J. L. I. 1988. Manejo de solos ácidos dos trópicos úmidos da região amazônica. Fundação Cargill, Campinas, 215p.

Falesi, I.C. 1976. Ecossistemas de pastagens cultivadas na Amazonia brasileira. Belèm, Embrapa/CPATU. , Belém, (Boletim Téenico 1). 193p.

Ferreira, M.E.; Souza, E.A.; Toledo Jr.; 
Kronka, S.N.; Aloisi, R.R. 1991. Influência do selênio e do cobre na determinação do nitrogênio total de solos. Encontro Regional da SBPC, Jaboticabal, v.1., p. 56.

Giulietti, A.M. 1992. Biodiversidade da região sudeste. Anais II Congresso Nacional sobre Essências Nativas, São Paulo, p. 125-130.

Hecht, S.B. 1983. Cattle ranching in eastern Amazonia. Berkeley, University of California (PhD Thesis). 455p.

Mello, F.A.F.; Brasil Sobrinho, M.O.C.; Arzolla, S.; Silveira, R.I.; Cobra Netto, A.; Kiehl, J.C. 1988. Fertilidade do solo, Nobel, São Paulo, 400p.

Melo, W.J. 1974. Variação do N-amonialcal e $\mathrm{N}$-nitrico em um latossolo roxo cultivado com milho (Zea mavs, L.) e com Lablab (Dolichos lablab). Piracicaba: Escola Superior de Agricultura "Luiz de Queiroz". (Tese de doutorado) 104p.

Moraes, J.F.L. 1991. Conteúdo de Carbono e Nitrogênio e tipologia nos solos da Bacia amazónica. CENA/USP. Piracicaba, (Dissertação de Mestrado). 84p.

Moraes, W.V. 1984. Comportamento de caracteristicas e propriedades fisicas de um latossolo vermelho-escuro submetido a diferentes sistemas de cultivo: ESAL, Lavras, (Dissertação de Mestrado). 207 p.

Nascimento, C.; Homma, A. 1984. A Amazónia: meio ambiente e tecnologia agricola. Embrapa/CPATU. Belém, 282p.

Nortcliff, S.; Thornes, J.B. 1982. Water and cation movement in a tropical rainforest environmental. Acta Amazonica, 8(2):245258.

Nye, P.H.; Greeland, D.J. 1964. Changes in the soil after clearing tropical forest. Plant and Soil, 21(2): 101-112.

Okali, D.U.U. 1992. Sustenable use of West African moist forest lands. Biotropica, 24: 335-344.
Prance G.T. 1987. Perdendo Rondônia. Rev. Cienc. Hoje, 6 (36): 48-56.

Primavesi, A. 1987. O manejo ecológico do solo. Nobel. São Paulo. 541p.

Radambrasil. 1983. Levantamento de recursos naturais. Ministério das Minas e Energia. Departamento Nacional de Produção Mineral, Rio de Janeiro, v.16 e 23.

Raij, B.V.; Quaggio, J.A.; Cantarella, H., Ferreira, M.E., Lopes, A.S., Bataglai, O.C. 1987. Análise quimica do solo para fins de fertilidade. Fundação Cargil. Campinas, SP. 170p.

Sanches, P.A. 1976. Properties and management of soils in the tropics. New York, Wiley. 619p.

Serrão, E.A.S.; Falesi, I.C.; Veiga, J.B. Teixeira, J.F. 1979. Productivity of cultivated pastures in low fertility soil of the amazon of Brazil. In: Sanches, P.A.; Tergas, L.E. (eds.) Pasture production in acid soils of the tropics. Cali, Colombia, CIAT, p. 195-226.

Serrão, E.A.S.; Toledo, J.M. 1990. The search for sustainability in Amazon pastures. In: Anderson, A. B. (ed.) Alternatives to deforestation: steps toward sustainable utilization of amazon forest. New York, Columbia University Press, p.195-214.

Seubert, C.E.; Sanches, P.A.; Valverde, C. 1977. Effects of land clearing methods on soil properties of an ultisol and crop perfomance in the Amazon jungle of peru. Tropical Agriculture, 54: 307-321.

Smyth, T.J. 1996. Manejo da fertilidade do solo para produção sustentada de cultivos na Amazônia. In: O solo nos grandes domínios morfoclimáticos do Brasil e o desenvolvimento sustentado. Soc. Bras. de Cienc. do Solo, Viçosa, p. 71-93.

Sparovek, G. 1993. Amostragem e análise de dados edafoclimáticos de um remanescente florestal no municipio de Pindorama (SP). ESALQ/USP, Piracicaba, (Dissertação de Doutorado), $132 \mathrm{p}$. 